\title{
Increased Intake of Dietary Polyunsaturated Fat Does Not Promote Whole Body or Preferential Abdominal Fat Mass Loss in Overweight Adults
}

\author{
Sze-Yen Tan ${ }^{\mathrm{a}}$ Marijka Batterham ${ }^{\mathrm{b}} \quad$ Linda Tapsell $^{\mathrm{c}}$ \\ ${ }^{\text {a }}$ Department of Nutrition Science, Purdue University, West Lafayette, IN, USA \\ ${ }^{\text {b }}$ Statistical Consulting Service, \\ ${ }^{\circ}$ Smart Foods Centre, University of Wollongong, Australia
}

\section{Keywords}

Polyunsaturated fatty acids - Weight loss · Fat mass .

Visceral adipose tissue

\section{Summary}

Objective: There is evidence that increasing the proportion of polyunsaturated fatty acids (PUFA) in a diet can enhance the rate of fat oxidation acutely. Higher PUFA in a diet has also been associated with greater abdominal fat loss in longer term studies. This study aimed to investigate if higher PUFA intake would result in greater fat mass loss over a 12-week period, mainly from the abdominal region. Methods: Data at the 12-week time point from two weight loss studies, both comparing high PUFA versus low PUFA diets was, accessed for 141 overweight subjects from the same area. Specifically, data on anthropometric measurements (weight, height, BMI, body composition, waist and hip circumference, SAT, VAT) and dietary intake were analyzed. The relationship between fat mass and VAT changes was examined using a differential equation. Results: Energy consumption decreased significantly in both study groups. The low fat groups decreased total dietary fat while the HPUFA groups increased PUFA intake significantly. All anthropometric measurements reduced significantly over time but there was no difference between the two dietary groups. The relationship between fat mass and visceral adipose tissue (VAT) loss was allometric. Conclusion: A higher PUFA intake did not lead to greater fat mass loss and there was no additional preferential loss of VAT following higher PUFA consumption.

\section{Introduction}

Dietary fats are high in energy density [1], are the least preferred source of energy during fuel selection by the body $[2,3]$, and are oxidized inefficiently during overfeeding $[4,5]$. For these reasons they have been implicated in the etiology of the worldwide obesity epidemic. However, fats are crucial in maintaining some physiological functions such as maintaining cell structure and transportation of fat-soluble vitamins [6]. In addition, to fully eliminate fat from the diet is not possible as we consume whole foods which come from food sources containing various nutrients in different proportions. Therefore, it is advisable to recommend restricting dietary fat intake to a sensible level and at the same time include optimal fats within a recommended level of intake.

Not all dietary fats have the same physiological functions, and there is evidence that different fat subtypes are oxidized in a hierarchy in the human body, depending on their chemical structures. The carbon chain length $[7,8]$ and degree of saturation [7] of fatty acids determine both the rate and order in which fats are oxidized. In humans, this order is polyunsaturated fatty acids (linolenate 18:3n-3; PUFA) first, followed by monounsaturated fatty acids (oleate 18:1n-9; MUFA), and saturated fatty acids (stearate 18:0; SFA) last [9]. At a cellular level, PUFA has been observed to play a role in repartitioning metabolic fuels for storage and oxidation by down-regulating the transcription of lipogenic genes and up-regulating the genes that promote lipid oxidation and thermogenesis [10]. Indeed, in a whole of diet context $[11,12]$, studies have confirmed a greater fat-oxidizing effect acutely when the ratio of PUFA:SFA increases. Supplementation of the diet using fish oil (PUFA rich) has also been shown to increase fat oxidation $[13,14]$. Greater fat oxidation implies a lower retention in the

\section{KARGER \\ Fax +497614520714 \\ Information@Karger.de}

www.karger.com
(C) 2011 S. Karger GmbH, Freiburg

1662-4025/11/0045-0352\$38.00/0

Accessible online at:

www.karger.com/ofa
Sze-Yen Tan, PhD

Departments of Foods and Nutrition

Purdue University

Stone Hall, 700 W State Street, West Lafayette, IN 47907, USA

Tel: +1765 49-63607, Fax -40906

tan66@purdue.edu 
Table 1. Study arms of Trial A and Trial B studies

\begin{tabular}{llll}
\hline Trial A & & & \\
\hline Low PUFA & High PUFA & Low PUFA and low energy & High PUFA \& low energy \\
\hline Isocaloric low-fat diet with $20 \%$ & Isocaloric low-fat high PUFA & A diet similar to the low fat diet & A diet similar to the high PUFA \\
protein, $50 \%$ CHO, $30 \%$ fat $(5 \%$ & diet with $20 \%$ protein, $50 \%$ & but with a $500 \mathrm{kcal} / \mathrm{d}$ energy & diet but with a 500 kcal/d energy \\
PUFA, $15 \%$ MUFA, $10 \%$ SFA). & CHO, 30\% fat (10\% PUFA, & deficit. &
\end{tabular}

Trial B

Low PUFA

High PUFA

Isocaloric low-fat diet with $20 \%$ protein, $50 \% \mathrm{CHO}, 30 \%$ fat

(5\% PUFA, $15 \%$ MUFA, $10 \%$ SFA). This study arm is the equivalent

Isocaloric low-fat high PUFA diet with $20 \%$ protein, $50 \% \mathrm{CHO}$,

of the low fat arm in Trial A.

$30 \%$ fat (10\% PUFA, $10 \%$ MUFA, $10 \%$ SFA). This study arm is the

equivalent of the high PUFA arm in Trial A.

$\mathrm{CHO}=$ Carbohydrate PUFA $=$ polyunsaturated fat MUFA = monounsaturated fat SFA = saturated fat.

body. This differential effect of fat subtypes means that the incorporation of PUFA into a diet may be beneficial in the promotion of negative fat balance and leads to the use of endogenous fat as energy source. It may be an important consideration in weight management where adipose tissue accumulation is integral to the problem.

Nonetheless, these observations were obtained from studies using an acute feeding design, and it remains unclear if an enhanced fat oxidation rate can be extended over a longer period of time in a free-living environment. The intriguing question is whether or not the increased fat oxidation rate is sufficient to induce a state of negative fat balance and cumulatively lead to greater fat mass (FM) loss in overweight individuals. Studies including a higher PUFA intake have been associated with a greater FM loss in the abdominal region when the intake of PUFA was increased $[15,16]$. This is beneficial to overweight individuals as a greater reduction in abdominal FM will lead to improved metabolic fitness and reduced risks of metabolic disease $[17,18]$. This is particularly the case if the loss of FM comes from the visceral adipose tissues (VAT). Hallgreen and Hall [19] analyzed data from weight loss trials that employed various methods (calorie restriction, exercise, and surgery) and found no preferential loss of VAT induced by any of the three methods or their combinations. They concluded that VAT loss is therefore proportionate to the amount of total body FM loss. However, studies using high PUFA diets were not included in their analysis. Therefore, this study aimed to investigate if a higher PUFA intake would result in greater FM loss in the short term, mainly from the abdominal region.

\section{Participants and Methods}

\section{Experimental Protocol}

Two dietary interventions (Trial A [20] and Trial B [21]) from which data was extracted had similar experimental protocols. The key intervention for both trials was increased dietary PUFA intake. Trial A was a 12-week randomized controlled trial with four study arms while Trial B was a 52-week randomized controlled trial with two study arms. The study arms are summarized in table 1.

Participants from both trials were recruited from the local community through media advertisement. Eligible participants attended a pre-study assessment session where anthropometric measurements, blood biochemistry, habitual dietary intake, and physical activity were assessed. In both trials, participants randomized into the high PUFA arms received structured dietary advice to incorporate PUFA-rich foods to achieve PUFA target of $10 \%$ energy. The intervention group in Trial B also received a daily supplement of $30 \mathrm{~g}$ walnuts. All participants received monthly supports from qualified dietitians to help adhere to the prescribed diets for the first 12 weeks. After the 12-week period, participants from Trial B attended quarterly follow-up sessions. The experiment protocols from both trials were approved by the University of Wollongong Human Research Ethics Committee and were registered with the Australian Clinical Trial Registry (Trial A: ACTRN12608000453381; Trial B: ACTRN12607000600448)

\section{Participants}

Inclusion criteria for Trial A [20] and Trial B [21] were age $>18$ years and BMI $>25 \mathrm{kgm}^{-2}$ (participants of both sexes were included); Trial B also included individuals who were diagnosed with type 2 diabetes (without insulin treatment). Participants were excluded if they have major illness, were smokers, allergic to factors inhibiting the trials, illiterate, or showed inadequate conversational English as dietary counseling was the key component of the intervention. Eligible participants were randomized into one of the study arms using random permuted blocks by a computerized random number generator. 20 participants were required in each arm to provide a power of $80 \%$ in order to detect a change of $20 \%$ in VAT, as reported by a previous study [15].

\section{Dietary Prescriptions and Physical Activity Recommendations}

Dietary recommendations for both Trial A and Trial B were based on core food groups to meet the target dietary nutrient composition profile of the different test diets. All participants received individualized [20,21] dietary counseling and had the same amount of contact (monthly) with qualified dietitians for the first 12 weeks of the studies. Participants in the high PUFA (HPUFA) arms were asked to include PUFA-rich foods in Trial A while participants in Trial B received a daily supply of walnuts ( $30 \mathrm{~g} /$ day) to help achieve PUFA target. Compliance to walnut consumption was confirmed through the significant increase in erythrocyte membrane $\alpha$-linoleic acid in the HPUFA group from baseline to 12 weeks [21]. Participants were also advised to maintain $5 \times 30$-min physical activity per week 
Table 2. Mean ( \pm SD) anthropometric measurements and dietary intakes at baseline and after 12 weeks

\begin{tabular}{|c|c|c|c|c|c|c|c|}
\hline & \multicolumn{2}{|l|}{ Baseline } & \multicolumn{2}{|l|}{12 weeks } & \multirow{2}{*}{$\begin{array}{l}\text { Time } \\
\text { effects } \\
\mathrm{p}\end{array}$} & \multirow{2}{*}{$\begin{array}{l}\text { Diet } \\
\text { effects } \\
\mathrm{p}\end{array}$} & \multirow{2}{*}{$\begin{array}{l}\text { Time } \\
\times \text { diet effects } \\
\mathrm{p}\end{array}$} \\
\hline & $\begin{array}{l}\text { LPUFA } \\
(\mathrm{n}=70)\end{array}$ & $\begin{array}{l}\text { HPUFA } \\
(\mathrm{n}=71)\end{array}$ & $\begin{array}{l}\text { LPUFA } \\
(\mathrm{n}=70)\end{array}$ & $\begin{array}{l}\text { HPUFA } \\
(\mathrm{n}=71)\end{array}$ & & & \\
\hline Weight, kg & $90.8 \pm 14.8$ & $88.4 \pm 13.9$ & $85.1 \pm 13.7$ & $84.0 \pm 14.7$ & $0.000^{*}$ & 0.464 & 0.859 \\
\hline BMI, $\mathrm{kg} / \mathrm{m}^{2}$ & $32.1 \pm 4.0$ & $31.4 \pm 3.9$ & $30.2 \pm 4.0$ & $29.9 \pm 4.4$ & $0.000 *$ & 0.463 & 0.844 \\
\hline Body fat, \% & $39.2 \pm 6.6$ & $39.4 \pm 7.0$ & $35.8 \pm 7.0$ & $37.1 \pm 8.3$ & $0.000 *$ & 0.799 & 0.177 \\
\hline VAT area, $\mathrm{cm}^{2}$ & $156 \pm 91$ & $141 \pm 81$ & $130 \pm 78$ & $131 \pm 90$ & $0.000 *$ & 0.606 & 0.052 \\
\hline $\mathrm{SAT}$ area, $\mathrm{cm}^{2}$ & $312 \pm 102$ & $338 \pm 107$ & $302 \pm 108$ & $316 \pm 113$ & $0.001^{*}$ & 0.198 & 0.179 \\
\hline EI, kcal/day & $2,181 \pm 694$ & $2,270 \pm 744$ & $1,754 \pm 514$ & $1,840 \pm 483$ & $0.000 *$ & 0.243 & 0.639 \\
\hline CHO, \%EI & $42.0 \pm 7.0$ & $43.8 \pm 8.7$ & $44.1 \pm 6.7$ & $43.1 \pm 6.3$ & $0.046^{*}$ & 0.804 & $0.035^{*}$ \\
\hline Protein, \%EI & $20.3 \pm 3.6$ & $19.7 \pm 3.7$ & $21.9 \pm 3.7$ & $21.0 \pm 3.5$ & $0.000 *$ & 0.066 & 0.957 \\
\hline Fat, \%EI & $31.7 \pm 6.1$ & $31.7 \pm 7.2$ & $27.7 \pm 5.9$ & $30.9 \pm 5.9$ & $0.000 *$ & $0.018^{*}$ & 0.066 \\
\hline MUFA, \%EI & $12.3 \pm 3.1$ & $12.4 \pm 4.3$ & $8.0 \pm 4.0$ & $7.6 \pm 3.8$ & $0.000 *$ & 0.909 & 0.242 \\
\hline PUFA, \%EI & $5.5 \pm 2.1$ & $5.1 \pm 2.0$ & $5.4 \pm 2.0$ & $10.2 \pm 3.6$ & $0.000 *$ & $0.000^{*}$ & $0.000 *$ \\
\hline
\end{tabular}

LPUFA = Low PUFA group; HPUFA = high PUFA group ; VAT = visceral adipose tissue; SAT = subcutaneous adipose tissue; EI = energy intake; $\mathrm{CHO}=$ carbohydrate; PUFA = polyunsaturated fat; MUFA = monounsaturated fat; SFA = saturated fat.

*Significant different, general linear model repeated measures ANOVA, $\mathrm{p}<0.05$.

\section{Measurements}

\section{Body Weight and Body Composition}

Body weight was measured, and percentage body fat was estimated using scales with bioelectrical impedance (Tanita TBF622; Tanita Corp., Tokyo, Japan) in an upright position with minimal clothing. Body fat measurements from this device have been previously shown to be comparable to those from the DEXA scans [22]. Single-slice abdominal computed tomography (CT) scans were also taken at the fourth and fifth lumbar vertebra (L4-L5), as well as at the level of sacroiliac joints by a trained observer at a commercial X-ray facility in Wollongong (Southcoast X-Ray, NSW, Australia). Subcutaneous adipose tissue (SAT) and VAT areas at L4-L5 levels were estimated using SIENET Sky software (Siemens AG, Munich, Germany), and the mean of these two levels were calculated. Using the mean VAT areas $\left(\mathrm{A}_{\mathrm{VAT}}\right)$, total volume of VAT $\left(\mathrm{V}_{\mathrm{VAT}}\right)$ at the abdominal region was estimated using the equations described by Shen and colleagues [23]:

Men: $\mathrm{V}_{\mathrm{VAT}}=0.0224 \times \mathrm{A}_{\mathrm{VAT}}+0.1621$

Women: $\mathrm{V}_{\mathrm{VAT}}=0.0205 \times \mathrm{A}_{\mathrm{VAT}}+0.1471$

\section{Relationship between FM and VAT Loss}

To examine if there is preferential loss of VAT, its changes will be examined alongside total FM loss using the mathematical model described by Hallgreen and Hall [19], which was an extension to the observations by the original works by Smith and Zachwieja [24]. It was hypothesized that FM and VAT changes follow an allometric relationship as shown by the differential equation below:

\section{$\mathrm{VAT} / \mathrm{FM}=\mathrm{k} \times \mathrm{dVAT} / \mathrm{dFM}$}

where VAT and FM refer to initial (before treatment) VAT and FM; dVAT and dFM refer to changes in VAT and FM over time; and $\mathrm{k}$ is the dimensionless constant which can be calculated from the other 4 known variables.

\section{Dietary Intake}

Habitual dietary intake was assessed using a validated diet history interview [25] conducted by dietitians and a 3-day food record. Dietary composition was analyzed using the FoodWorks nutrient analysis software package (Xyris Software, Professional Version 2002, Brisbane, Australia). Fatty acid intake profiles were obtained using the AUSNUT fatty acid database (Version 6, 2002) in the FoodWorks software package and the Australian Fatty Acids Rev 62002 (RMIT, Melbourne, Australia).

\section{Data Analysis}

For the purpose of this paper, only data collected at baseline and 12 weeks was included. Data from Trial A and Trial B were combined in order to test the relationship between FM and VAT changes. The study arms in both studies were collapsed into low fat (LPUFA) and HPUFA groups for comparison purposes. Data was analyzed using SPSS statistical software (version 15.0.0, SPSS, Chicago, IL, USA). Baseline differences between diet groups were assessed using one-way ANOVA, with post hoc comparisons using a Bonferroni adjustment. Changes in the primary outcomes between LPUFA and HPUFA were compared using the general linear model for repeated measures ANOVA.

\section{Results}

\section{Participants}

From both Trial A and Trial B, 273 participants were screened, where 200 were eligible and enrolled. They were randomly assigned to one of the study arms. 28 volunteers withdrew prior to the commencement of the studies (Trial A, $\mathrm{n}=18$; Trial $\mathrm{B}, \mathrm{n}=10$ ), and 31 were lost to follow-up visits (Trial $A, n=11$; Trial $B, n=20$ ). Of the original 200 participants, 141 completed the studies. Data from 121 out of the 
Table 3. Characteristics of participants $(\mathrm{N}=121)$ at baseline and 12 -week of study

\begin{tabular}{|c|c|c|c|c|c|c|c|}
\hline & \multicolumn{2}{|l|}{ Baseline } & \multicolumn{2}{|l|}{12 weeks } & \multirow{2}{*}{$\begin{array}{l}\text { Time } \\
\text { effects } \\
\mathrm{p}\end{array}$} & \multirow{2}{*}{$\begin{array}{l}\text { Diet } \\
\text { effects } \\
\mathrm{p}\end{array}$} & \multirow{2}{*}{$\begin{array}{l}\text { Time } \\
\times \text { diet effects } \\
\mathrm{p}\end{array}$} \\
\hline & $\begin{array}{l}\text { LPUFA } \\
(\mathrm{n}=58)\end{array}$ & $\begin{array}{l}\text { HPUFA } \\
(n=63)\end{array}$ & $\begin{array}{l}\text { LPUFA } \\
(\mathrm{n}=58)\end{array}$ & $\begin{array}{l}\text { HPUFA } \\
(n=63)\end{array}$ & & & \\
\hline Age, years & $48.4 \pm 10.6$ & $47.6 \pm 10.5$ & - & - & - & - & - \\
\hline Weight, kg & $88.0 \pm 13.7$ & $87.2 \pm 14.0$ & $84.7 \pm 13.7$ & $83.7 \pm 14.6$ & $0.000 *$ & 0.721 & 0.803 \\
\hline BMI, $\mathrm{kg} / \mathrm{m}^{2}$ & $31.3 \pm 3.8$ & $31.0 \pm 4.0$ & $30.1 \pm 4.0$ & $29.8 \pm 4.4$ & $0.000 *$ & 0.657 & 0.791 \\
\hline FM, kg & $33.6 \pm 8.2$ & $33.9 \pm 9.0$ & $30.6 \pm 8.2$ & $31.3 \pm 9.7$ & $0.000^{*}$ & 0.753 & 0.376 \\
\hline FFM, kg & $54.4 \pm 9.9$ & $53.3 \pm 10.3$ & $54.1 \pm 10.2$ & $52.4 \pm 10.4$ & $0.000 *$ & 0.449 & $0.023 *$ \\
\hline $\mathrm{V}_{\mathrm{VAT}}, 1$ & $3.5 \pm 2.0$ & $3.2 \pm 1.8$ & $2.9 \pm 1.7$ & $2.9 \pm 2.0$ & $0.000 *$ & 0.628 & 0.065 \\
\hline
\end{tabular}

LPUFA = Low PUFA group; HPUFA = high PUFA group; FM = fat mass; FFM = fat-free mass; VAT = visceral adipose tissue.

*Significant different, general linear model repeated measures ANOVA.

141 subjects was included because CT scans at both time points were available. 20 subjects did not have the CT scan done at 12 weeks of the studies, and data from these subjects were excluded. Baseline characteristics and habitual intake of these participants were not statistically different $(p>0.05)$ (table 2).

\section{Dietary Change}

Dietary intervention effect was achieved in Trial A and Trial B. The combined analysis for the purpose of this paper shows that the intakes of energy and macronutrients changed significantly within each study group (table 2) after the 12-week intervention period. Of all macronutrients, only the percentages of total fat (diet, $\mathrm{p}=0.018$ ) and PUFA (diet, $\mathrm{p}<0.001$ ) were significantly different between groups, where the LPUFA group reduced fat intake and the HPUFA group increased PUFA consumption. The interaction effects were significant for carbohydrate $(p=0.035)$, SFA $(p=0.003)$ and PUFA $(\mathrm{p}<0.001)$ proportions in the diets. The LPUFA group increased the proportion of carbohydrate to substitute dietary fat while the HPUFA group increased their PUFA intake at the expense of SFA in the diet.

\section{Anthropometric Change}

Weight change was significant in the original studies (Trial A and Trial B), and when data was combined and analyzed in this study, all groups also lost weight significantly after the 12 -week period. Subsequently, all other parameters were significantly reduced over time (time, $\mathrm{p} \leq 0.001$ ). However, these changes were not different between the LPUFA and HPUFA groups, and interaction effects were also not observed (table 2).

\section{Relationship between FM and VAT Changes}

To test if the relationship between the changes of these two variables was allometric, 121 (58 LPUFA and 63 HPUFA) complete data sets containing FM and VAT volume from Trial A and Trial B were analyzed. Baseline and post-intervention (12-week) measurements of weight, BMI, FM, fatfree mass (FFM), and VAT volume are presented in table 3.

FM and volume of VAT decreased significantly in both groups after the 12-week period, but there was no difference between LPUFA and HPUFA groups. The dimensionless constant was $\mathrm{k}=1.15 \pm 3.59$ for the LPUFA group and $\mathrm{k}=1.23 \pm 2.86$ for the HPUFA group, and they were not significantly different between groups (independent sample t-test, $\mathrm{p}=0.896)$.

\section{Discussion}

This study aimed to investigate the relationship between dietary PUFA intake, FM loss as well as preferential abdominal FM loss as reported by other studies. Using the data from two intervention trials, we showed that the enhanced fat oxidation by PUFA observed in acute studies failed to produce greater FM loss using a longitudinal study design. Previous studies reported abdominal fat loss and total FM loss independently; when we examined them together, preferential loss of abdominal fat following increased PUFA intake was not observed.

There were some limitations to this study. It included convenient samples obtained from two separate clinical trials that utilized different dietary strategies. However, the treatments and study protocols were similar (high vs. low PUFA intake), and the clinical outcomes were not likely to be confounded by these differences. This study compared data between baseline and 12 weeks only, making the comparison over a longer period of time impossible. This was due to the length of original study (Trial A), but 3-month changes in weight and body composition are still of clinical importance. There was also concern over the PUFA consumptions in both study groups, where some participants in LPUFA group consumed higher 
PUFA while some in the HPUFA group consumed low PUFA during the data collection period. When we performed head-to-head comparison, the change in PUFA intake and FM (0-12 weeks) did not correlate for all participants ( $\mathrm{r}=$ $0.076, p=0.409)$. It turns out that this analysis further supports and strengthens our observation that changing PUFA intake may have limited impact on body FM. Physical activity was not measured during the intervention period, and it was uncertain if this has changed. Precautions were taken in the original trials where participants were told to maintain 30-min exercise five times a week, and this was emphasized by the dietitians on a monthly basis.

This study also has a number of strengths. First, it examines the possibilities of acute metabolic effects of increased PUFA intake observed in a controlled laboratory setting be extended into a less-restrictive free-living environment over a 12 -week period. This is crucial in demonstrating the effectiveness and practicality of a weight management strategy. Another strength of this study is that it differs from the previous studies in that it compares the change in visceral fat relative to the total fat change, not in isolation. Pooling data from our two previous studies that originally addressed other specific study outcomes provided us with a greater sample size to examine this issue. This study points out errors in conclusions that can be drawn if FM and VAT are considered independently. For example, although the between-groups differences in VAT area and volume were approaching statistical significance $(\mathrm{p}=0.052$ and $\mathrm{p}=0.065$, respectively), they diminished $(p=0.896)$ when examined as a ratio to total FM change.

The failure to replicate the results from previous studies was not likely to be confounded by the dietary compliance in this study, where dietary effects were reported in the original trials, and the combined data. Diet history analyses showed that the HPUFA groups increased their PUFA proportion in the diet while the LPUFA groups did not change their PUFA consumption after 12 weeks, indicating effective and successful execution of dietary interventions. One can increase the intake of PUFA by substituting SFA either through careful dietary modeling to identify and incorporate PUFA-rich foods into the diet or by supplementing with foods like walnuts.

This study was motivated by previous research that reported a higher thermogenic effect of PUFA in the short term, where the basal metabolic rate and thermic effect of food were observed to elevate following meals with higher PUFA content [26, 27]. However, this was not reflected in our study as the weight loss of participants in the HPUFA group was not higher than that in the LPUFA group, although both groups lost weight significantly over the 12 -week study period. Weight loss is likely to largely result from the significantly lower energy intake after 12 weeks as shown in table 2 . This implies that either the elevated thermogenesis was not great enough to produce a significantly greater weight loss or the thermogenic effect of HPUFA diets has been washed out in a free-living environment. These are the areas that warrant further investigation.

Changes in FM in our analysis follow a similar trend to weight loss with a significant FM loss over time, but no diet effect was observed. Although there is indication that higher PUFA consumption acutely enhanced fat oxidation [9], our data suggests that this acute effect could not be translated into weight loss in the longer term in a free-living environment. Our observation is not consistent with the results of a study by Couet and colleagues [14] who found significantly greater FM loss over a 3-week period. This may be due to the differences in a few aspects of the study. Overweight participants in our study may have different fat oxidation rates to the lean subjects in study by Couet et al. [28], where overweight individuals have been shown to have blunted lipid oxidation. The difference in the length of study and the use of supplements and how they are digested and metabolized may impact on the compliance to the treatment. At this stage, the effects of adaptations of the human body to increased dietary PUFA remains unclear.There was a significant interaction effect of treatment on FFM changes in our study, where the HPUFA group lost more FFM than the LPUFA group $(-0.3 \mathrm{~kg} / 12$ weeks vs. $-0.9 \mathrm{~kg} / 12$ weeks). The significant change in FFM was a novel observation because limited studies have compared the effectiveness of high versus low PUFA diets in promoting weight and FM loss. From a mechanistic perspective, it may be speculated that higher PUFA intake increases skeletal muscle membrane PUFA composition, which has been reported to be inversely correlated with fasting insulin level [29]. Indeed, a significantly greater decrease in fasting insulin in the HPUFA group was observed in Trial $B$ [21]. Insulin is an anabolic hormone, and its decrease related to increased PUFA intake may have contributed to the greater FFM loss in the HPUFA arm in this study. Further investigations are required to explain such observation since empirical data to support this speculation is lacking.

The current analysis did not demonstrate additional benefits of a HPUFA diet on abdominal fat loss. This was evident from the similar changes in SAT and VAT areas in both study groups. When VAT area was converted into VAT volume, changes in VAT were again not significantly different between groups. The mean $\mathrm{k}$ values, calculated using equation (3), as outlined in the methods section, were very close to those reported by Hallgreen and Hall [19], where studies using energy restriction alone produced a mean value of $\mathrm{k}=1.2$ while combined strategies involving energy restriction and resistance exercise produced $\mathrm{k}=1.3$. Effects of LPUFA diet in this study generated a $\mathrm{k}$ value close to energy restriction (mean $\mathrm{k}=1.15$ ), while $\mathrm{k}$ of HPUFA fell between energy restriction with and without resistance exercise (mean $\mathrm{k}=1.23$ ). Nonetheless, dimensionless constant $\mathrm{k}$ was not different between LPUFA and HPUFA groups, implying that there is preferential loss of VAT $(\mathrm{k}>1)$, but it was not different from VAT loss induced by other weight loss strategies. 


\section{Conclusion}

Energy restriction produced greater weight loss with or without increasing PUFA in the diet. The HPUFA diet failed to produce greater FM loss despite greater fat oxidation rates observed in other acute feeding trials. The dimensionless constant, $\mathrm{k}$, was also not different, implying that there was no preferential fat oxidation in the abdominal region from the higher PUFA intake. This study suggests that the loss of VAT depends on the total loss of body FM and is minimally influenced by the PUFA in the diet.

\section{Acknowledgements}

The authors would like to acknowledge the National Health and Medical Research Council of Australia (NHMRC) for funding Trial A and the Californian Walnut Commission USA (CWC) for Trial B.

\section{Disclosure Statement}

The authors declared no conflict of interest.

\section{References}

1 Widdowson EM: Assessment of the Energy Value of Human Foods. London, Cambridge University Press, 1955.

2 Bickerton AS, Roberts R, Fielding BA, Hodson L, Blaak EE, Wagenmakers AJM, Gilbert M, Karpe F, Frayn KN: Preferential uptake of dietary fatty acids in adipose tissue and muscle in the postprandial period. Diabetes 2007;56:168-176.

3 Stubbs J, Raben A, Westerterp-Plantenga MS: Macronutrient metabolism and appetite; in Westerterp-Plantenga MS, Steffens AB, Tremblay A (eds): Regulation of Foods Intake and Energy Expenditure. Milan, Edra, 1999, pp 59-84.

$\checkmark 4$ Horton TJ, Drougas H, Brachey A, Reed GW, Peters JC, Hill JO: Fat and carbohydrate overfeeding in humans: different effects on energy storage. Am J Clin Nutr 1995;62:19-29.

$\checkmark 5$ Astrup A, Buemann B, Christensen NJ, Toubro $\mathrm{S}$ : Failure to increase lipid oxidation in response to increasing dietary fat content in formerly obese women. Am J Physiol Endocrinol Metabol 1994; 266:E592-599.

6 Guthrie HA, Picciano MF: Human Nutrition. St. Louise, Mosby, 1995.

7 Leyton J, Drury PJ, Crawford MA: Differential oxidation of saturated and unsaturated fatty acids in vivo in the rat. Br J Nutr 1987;57:383-393.

$\checkmark 8$ Cenedella RJ, Allen A: Differences between the metabolism of linoleic and palmitic acid: utilization for cholesterol synthesis and oxidation to respiratory $\mathrm{CO}_{2}$. Lipids 1969;4:155-158.

$\checkmark$ DeLany JP, Windhauser MM, Champagne CM, Bray GA: Differential oxidation of individual dietary fatty acids in humans. Am J Clin Nutr 2000;72: 905-911.

10 Clarke SD: Polyunsaturated fatty acid regulation of gene transcription: a mechanism to improve energy balance and insulin resistance. Br J Nutr 2000;83: S59-S66.

11 Jones PJH, Ridgen JE, Phang PT, Birmingham CL: Influence of dietary fat polyunsaturated to saturated ratio on energy substrate utilization in obesity. Metabolism 1992;41:396-401.
12 Jones PJH, Schoeller DA: Polyunsaturated: saturated ratio of diet fat influences energy substrate utilization in the human. Metabolism 1988;37:145151.

13 Delarue J, Couet C, Cohen R, Brechot J-F, Antoine J-M, Lamisse F: Effects of fish oil on metabolic responses to oral fructose and glucose loads in healthy humans. Am J Physiol Endocrinol Metabol 1996;270:E353-E362.

14 Couet C, Delarue J, Ritz P, Antoine J-M, Lamisse F: Effect of dietary fish oil on body fat mass and basal fat oxidation in healthy adults. Int $\mathrm{J}$ Obes 1997;21:637-643.

15 Summers LKM, Fielding BA, Bradshaw HA, Ilic V, Beysen C, Clark ML, Moore NR, Frayn KN: Substituting dietary saturated fat with polyunsaturated fat changes abdominal fat distribution and improves insulin sensitivity. Diabetologia 2002;45: 369-377.

16 O'Dea K, Walker KZ: Dietary composition can influence patterns of regional fat loss. Aus J Nutr Diet 1998;55:S32-S36.

17 Fox CS, Massaro JM, Hoffmann U, Pou KM, Maurovich-Hovart P, Liu CY, Vasan RS, Murabito JM, Meigs JB, Cupples LA, D'Agostino RB, O'Donnell CJ: Abdominal visceral and subcutaneous adipose tissue compartments: association with metabolic risk factors in the Framingham Heart Study. Circulation 2007:116:39-48.

18 Wajchenberg BL: Subcutaneous and visceral adipose tissue: their relation to the metabolic syndrome. Endocr Rev 2000;21:697-738.

19 Hallgreen CE, Hall KD: Allometric relationship between changes of visceral fat and total fat mass. Int J Obes 2008;32:845-852.

20 Tapsell LC, Batterham MJ, Huang XF, Tan SY, Teuss G, Charlton K, O'Shea J, Warensjo E: Short term effects of energy restriction and dietary fat sub-type on weight loss and disease risk factors. Nutr Metab Cardiovasc Dis 2010;20:317-325.
21 Tapsell LC, Batterham MJ, Teuss G, Tan SY, Dalton S, Quick CJ, Gillen LJ, Charlton K: Long-term effects of increased dietary polyunsaturated fat from walnuts on metabolic parameters in type 2 diabetes. Eur J Clin Nutr 2009;63:1008-1015.

22 Batterham MJ, Tapsell LC, Jenkins AB: A comparison of bioelectrical impedance and near infrared interactance with dual energy x-ray absorptiometry for the determination of body fat. Nutr Diet 2002;59:120-126.

23 Shen W, Punyanitya M, Wang ZM, Gallagher D, St-Onge M-P, Albu J, Heymsfield SB, Heshka S: Visceral adipose tissue: relations between singleslice areas and total volume. Am J Clin Nutr 2004; 80:271-278.

24 Smith SR, Zachwieja JJ: Visceral adipose tissue: a critical review of intervention strategies. Int J Obes Relat Metab Disord 1999;23:329-335.

-25 Martin GS, Tapsell LC, Denmeade SL, Batterham MJ, Russell KG: Relative validity of diet history interview in an interventional trial manipulating dietary fat in the management of type II diabetes mellitus. Prev Med 2003;36:420-428.

26 Casas-Agustench P, Lopez-Urirte P, Bullo M, Ros E, Gomez-Flores A, Salas-Salvado J: Acute effects of 3 high-fat meals with different saturation on energy expenditure, substrate oxidation and satiety. Clin Nutr 2009;28:39-45.

27 van Marken Lichtenbelt WD, Mensink RP, Westerterp KR: The effect of fat composition of the diet on energy metabolism. Zeitschr Ernährungswissensch 1997;36:303-305.

28 Kim J-Y, Hickner RC, Cortright RL, Dohm GL, Houmard JA: Lipid oxidation is reduced in obese human skeletal muscle. Am J Physiol Endocrinol Metabol 2000;279:E1039-E1044.

29 Storlien LH, Pan DA, Kriketos AD, O‘Connor J, Caterson ID, Cooney GJ, Jenkins AJ, Baur LA: Skeletal muscle membrane lipids and insulin resistance. Lipids 1996;31:S261-S265. 